\title{
Bilateral translaminar osseous-channel assisted percutaneous full-endoscopic ligamentectomy decompression for thoracic myelopathy due to ossification of the ligamentum flavum: a technical note
}

\author{
Zhijun Xin, Weijun Kong, Qian Du, Wenbo Liao \\ Department of Orthopaedic Surgery, Affiliated Hospital of Zunyi Medical University, Zunyi, Guizhou, China
}

Videosurgery Miniinv 2021; 16 (2): 429-441

DOI: https://doi.org/10.5114/wiitm.2020.100719

\begin{abstract}
Introduction: A variety of surgical procedures have been introduced to treat patients with thoracic myelopathy (TM) caused by ossification of the ligamentum flavum (OLF). However, they are accompanied by significant trauma and risk, and their surgical outcomes are not always satisfactory.

Aim: To describe a bilateral translaminar osseous-channel assisted posterior percutaneous full-endoscopic ligamentectomy decompression (p-PELD) technique as a novel minimally invasive procedure for treating patients with TM due to OLF.

Material and methods: A 51-year-old female patient with persistent thoracolumbar back pain and progressive numbness in the bilateral lower extremities for 2 years underwent percutaneous vertebroplasty (PVP) for T11 osteoporotic compression fractures (OCF) in a regional hospital one week prior to hospitalization. TM caused by canal stenosis and dorsal spinal cord compression at T10/11 secondary to OLF and an OCF at T11 were diagnosed based on clinical presentations and radiologic examinations. After bilateral p-PELD for TM was performed at T10/11, the result was confirmed based on postoperative radiographic and clinical results.

Results: The bilateral OLF was completely removed by the p-PELD technique. No complications were encountered, and her symptoms were sufficiently improved after surgery. The VAS score was 6 points preoperatively and decreased to 0 points at the last follow-up (24 months). The modified Japanese Orthopaedic Association (mJOA) score improved from 2 points preoperatively to 10 points at the final follow-up.

Conclusions: As a minimally invasive technique, the bilateral translaminar osseous-channel assisted p-PELD procedure provided precise and sufficient decompression for the treatment of OLF-related TM.
\end{abstract}

Key words: bilateral translaminar osseous-channel, full-endoscopic ligamentectomy decompression, ossification of the ligamentum flavum, thoracic myelopathy.

\section{Introduction}

Ossification of the ligamentum flavum (OLF) is a well-known cause of thoracic spinal stenosis that has been identified as the primary cause of thoracic myelopathy (TM) [1-3]. Particularly in East Asian re- gions, the incidence of thoracic OLF can be as high as $36 \%$ in the Japanese population and $63.9 \%$ in the Chinese population, although most such cases do not necessarily develop into asymptomatic thoracic canal stenosis and TM [4]. Once the thoracic OLF is symp- 
tomatic, surgical intervention is recommended because it is refractory to conservative treatment $[5,6]$, and this approach also avoids acute myelopathy induced by a minor trauma [7]. Although several multiple decompression procedures with open approaches or tube techniques, including laminectomy [2], aminoplasty [8], with or without instrumented fusion [6], have been described, the optimal surgical procedure remains controversial [1,7], and surgical outcomes are not always satisfactory $[2,9]$. The major concerns include greater iatrogenic trauma [10], a higher risk of neurological deterioration and complications [5, 11], and whether instrumentation should be used after the decompression procedure $[6,12]$.

With the advancement of surgical techniques and instruments, good results and advantages have been described for full-endoscopic discectomy techniques for lumbar stenoses and cervical spondylosis

Table I. The modified Japanese Orthopaedic Association (mJOA) scoring system for thoracic myelopathy

\begin{tabular}{|ll|}
\hline Neurological status & Score \\
\hline Lower-limb motor dysfunction: & 0 \\
\hline Anable to walk & 1 \\
\hline Able to walk up/downstairs with handrail & 2 \\
\hline Lack of stability \& smooth reciprocation of gait & 3 \\
\hline No dysfunction & 4 \\
\hline Lower-limb sensory deficit: & 0 \\
\hline Severe sensory loss or pain & 1 \\
\hline Mild sensory deficit & 2 \\
\hline No deficit & 0 \\
\hline Trunk sensory deficit: & 1 \\
\hline Severe sensory loss or pain & 2 \\
\hline Mild sensory deficit & 3 \\
\hline No deficit & \\
\hline Sphincter dysfunction: & 2 \\
\hline Unable to void & \\
\hline Marked difficulty in micturation difficulty in micturation & \\
\hline
\end{tabular}

myelopathy [13-15]. However, few reports have explored the use of posterior percutaneous full-endoscopic ligamentectomy decompression ( $p$-PELD) for TM caused by OLF.

\section{Aim}

The objective of this study was to describe the rationale, surgical technique, and the results of a new minimally invasive spinal surgery (MISS) technique, bilateral translaminar osseous-channel assisted posterior $\mathrm{p}$-PELD, as a treatment for TM caused by OLF.

\section{Material and methods}

\section{History}

Additional informed consent was obtained from a 51-year female patient who was transferred to our hospital from a regional hospital after a percutaneous vertebroplasty (PVP) with methyl methacrylate inserted for T11 osteoporotic compression fracture (OCF). Prior to hospitalization, with denial of a traceable history of trauma, a 2-year history of progressive thoracolumbar back pain and numbness disturbance of the lower limbs was present. Progressive spastic paraparesis of the lower extremity was present for 3 months, and gait disturbance with bladder and bowel sphincter dysfunction was present for 2 weeks. A physical examination revealed hypoalgesia in the bilateral lower extremities and inguinal regions. In addition, diffuse weakness was observed in the bilateral lower extremities, with 4/5 strength reported in all muscle groups. With a bilateral positive Babinski sign, her patellar tendon reflex and Achilles' tendons reflex were also exaggerated. Even with no symptomatic deterioration on the fifth day after the initial PVP operation, there were still 6 points of thoracolumbar back pain residue according to a visual analogue scale (VAS) evaluation [16], and her score in the modified Japanese Orthopaedic Association (mJOA) scoring system (Table I) for TM was 2 out of 11 possible points [17]. Preoperative diagnostic imaging included plain radiography, computed tomography (CT), and magnetic resonance imaging (MRI), which were performed before the initial PVP operation. However, no postoperative control CT was performed between the vertebroplasty and the endoscopy. No evidence of instability was found on flexion and extension lateral radiographs. A CT scan demonstrated spinal canal stenosis at the 
T10/11 level due to the presence of OLF (Photo 1). An MRI revealed dorsal compression and myelomalacic changes of the spinal cord at the T10/11 level secondary to OLF (Photo 2).

\section{Surgical technique and intraoperative findings}

After a diagnosis of TM caused by OLF was made, bilateral translaminar osseous-channel assisted p-PELD for resection of the OLF was performed at the T10/11 level. After general anaesthesia and neurological monitoring were performed, the patient was placed in a prone position. Intraoperative radiography was used to confirm the involved segment, and the surface projection of the responsible OLF was marked on the skin with ink. A needle was inserted with a trajectory aimed at the laminar target position (beginning with the right side) where the OLF lay hidden under the monitor of a "C" arm X-ray machine. Then, a $6-\mathrm{mm}$ dilator was inserted along the guide needle onto the laminar target position to bluntly separate the muscles. Insertion of a trephine (OD = $7.5 \mathrm{~mm}, \mathrm{ID}=6.5 \mathrm{~mm}$, Joimax, Germany) was performed via the dilator, and punching was performed on the target position of the lamina. Then, we performed a clockwise rotation and forwarding accuracy depth (Photos $3 \mathrm{~A}, \mathrm{~B}$ ), which was measured using preoperative imaging of the vertebral lamina. Then, the exact resected area of the lamina was removed overall with the trephine (Photo $3 \mathrm{C}$ ), and a translaminar osseous channel was made to allow the working sheath access into the canal (Photos $3 \mathrm{D}, \mathrm{E})$.

Then, the endoscope was inserted through the working sheath, and the further operation was performed under visual control and continuous irrigation with $0.9 \%$ saline solution. Limited laminectomy and adequate resection of the OLF was performed using a 3-mm diameter endoscopic high-speed drill (Midas Rex; Medtronic, Fort Worth, Texas, Germany) under endoscopic visualization (Photo 4 A). In this procedure, decompression was performed only on the side ipsilateral to the ossification lesion by slightly tilting the working sheath in combination with a 30-degree endoscope to achieve accurate, adequate decompression of the target area (Photo 4B). Care was taken to reduce direct extrusion on the dural sac during the decompression process.

Full exposure to the lateral margin and medial part of the dural sac was applied to achieve sufficient

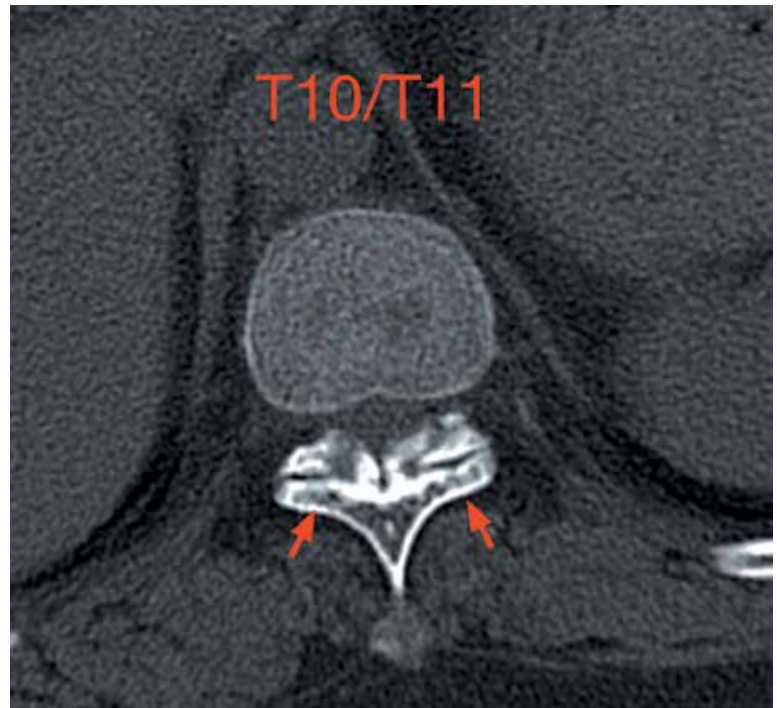

Photo 1. Preoperative CT scan. Preoperative axial CT scan performed before the initial PVP operation showing narrowing of the spinal canal at the T10/11 level due to the bilateral OLF (arrows)

decompression, and free-floating dura mater in the irrigation fluid was considered a sign of sufficient decompression. After adequate decompression was confirmed, a meticulous close suture of the soft tissue was performed without the use of drainage. Then, the same operation was performed on the left side.

There was no specific postoperative management. After bed rest for 1 day after surgery, the patient was discharged from the hospital on the third postoperative day and required to ambulate with assistance by a thoracolumbar orthotic brace for the next 3 months. Surgery-related indicators were collected, and radiological and clinical evaluations were performed preoperatively and at 1 week, 3 months, and 2 years postoperatively.

\section{Results}

After bilateral decompression at a single segment of the thoracic spine was successfully performed in this patient, her clinical symptoms improved, and she began to walk with the assistance of a unilateral crutch on the second postoperative day. The operation time was $109 \mathrm{~min}$ for the bilateral decompression, and there was little bleeding, which was estimated to be about $20 \mathrm{ml}$. During a 2-year follow-up period, no complications were observed in the patient. 

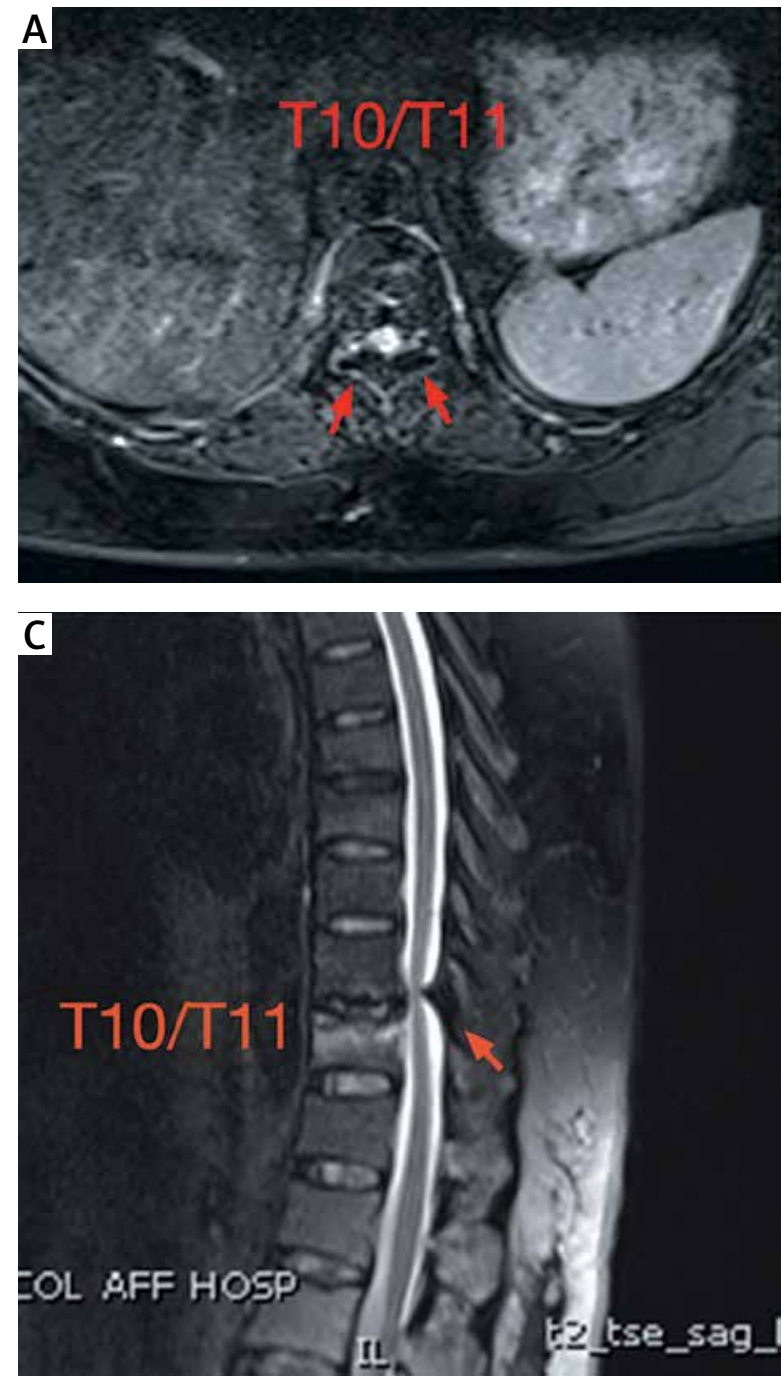

No evidence of instability or hypermobility was found at the T10/11 level on postoperative lateral dynamic radiographs (Photo 5), and adequate resection of the OLF and decompression of the spinal cord were observed on postoperative CT (Photo 6) and MRI (Photo 7).

The numbness of the bilateral lower extremity and inguinal regions was improved immediately after the operation, and the muscle strength of the lower extremities and sphincter continued to improve further with the passage of time. Generally, the patient's clinical outcome was good, including the VAS for thoracolumbar back pain that improved from preoperative 6 , to 3,1 , and 0 at 1 week, 6 months, and 2 years postoperatively, respectively, and the mJOA score for neurological status, which improved from preoperative 2 to 4,7 , and 10 at one

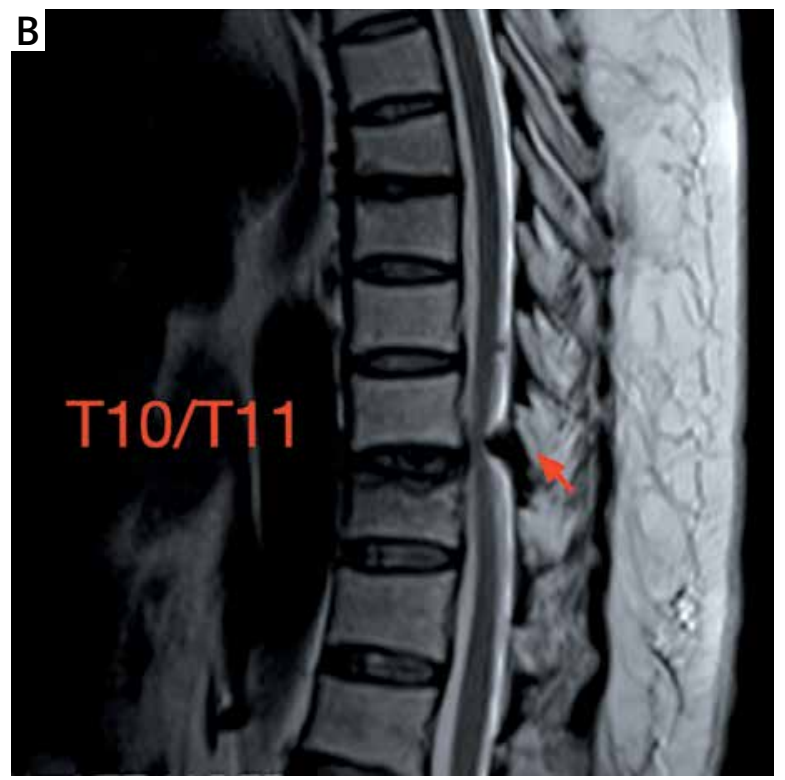

Photo 2. Preoperative MR images. Preoperative MR images performed before the initial PVP operation. A - Axial fat suppression MRI of the stenotic segment shows a nodular low-signal mass (OLF, arrows). Sagittal T2-weighted (B) and fat suppression (C) MRI showed dorsal spinal cord compression by low-signal masses (OLF, arrows) at the T10/11 level and an osteoporotic compression fracture of T11

week, 6 months, and 2 years postoperatively, respectively (Table II).

\section{Discussion}

As one of the primary causes of thoracic spinal stenosis and TM $[1,18]$, OLF has been shown to have a prevalence of $3.8 \%$ to $25 \%$ in previous studies [ 19 , 20]. With the improvement of diagnostic techniques and the increasing use of CT scanning and MRI, higher prevalence rates of OLF have been confirmed, including a rate as high as $63.9 \%$ in Chinese populations [21]. Although the pathological mechanism of OLF remains unclear, many conceivable risk factors have been proposed, such as mechanical, degenerative, metabolic, genetic, and dietary factors [22, 23]. However, there is still no effective way to prevent or 

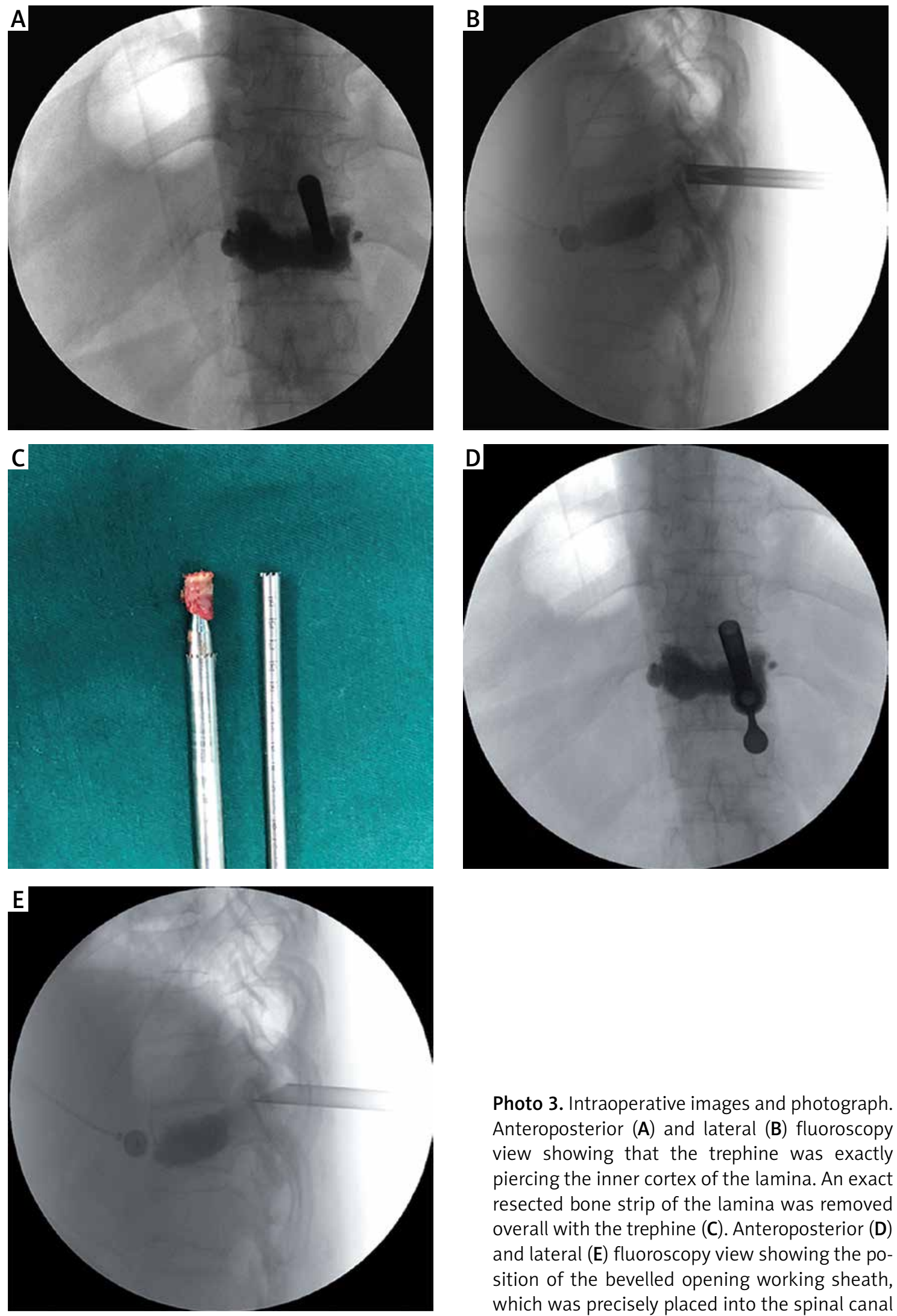

Photo 3. Intraoperative images and photograph. Anteroposterior (A) and lateral (B) fluoroscopy view showing that the trephine was exactly piercing the inner cortex of the lamina. An exact resected bone strip of the lamina was removed overall with the trephine (C). Anteroposterior (D) and lateral (E) fluoroscopy view showing the position of the bevelled opening working sheath, which was precisely placed into the spinal canal 

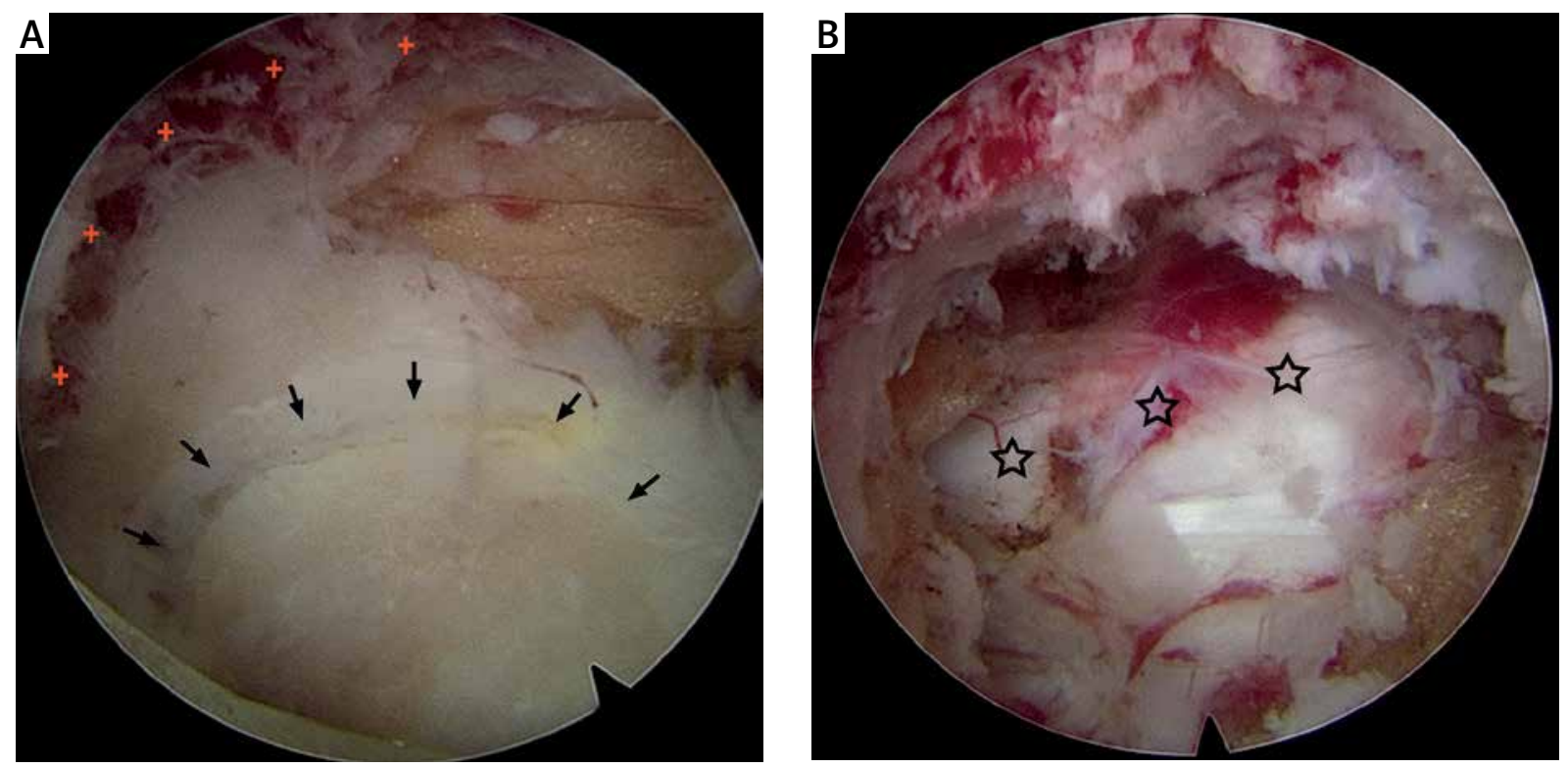

Photo 4. Endoscopic views. A - Intraoperative endoscopic view before decompression demonstrated a circular osteal groove (crosses) that was made by a trepan on the laminar and the ossified mass (OLF, arrows) presenting in the enlarged endoscopic visual field. B - Intraoperative endoscopic view after resection of the ossified mass (OLF) revealed the decompressed dural sac (stars)
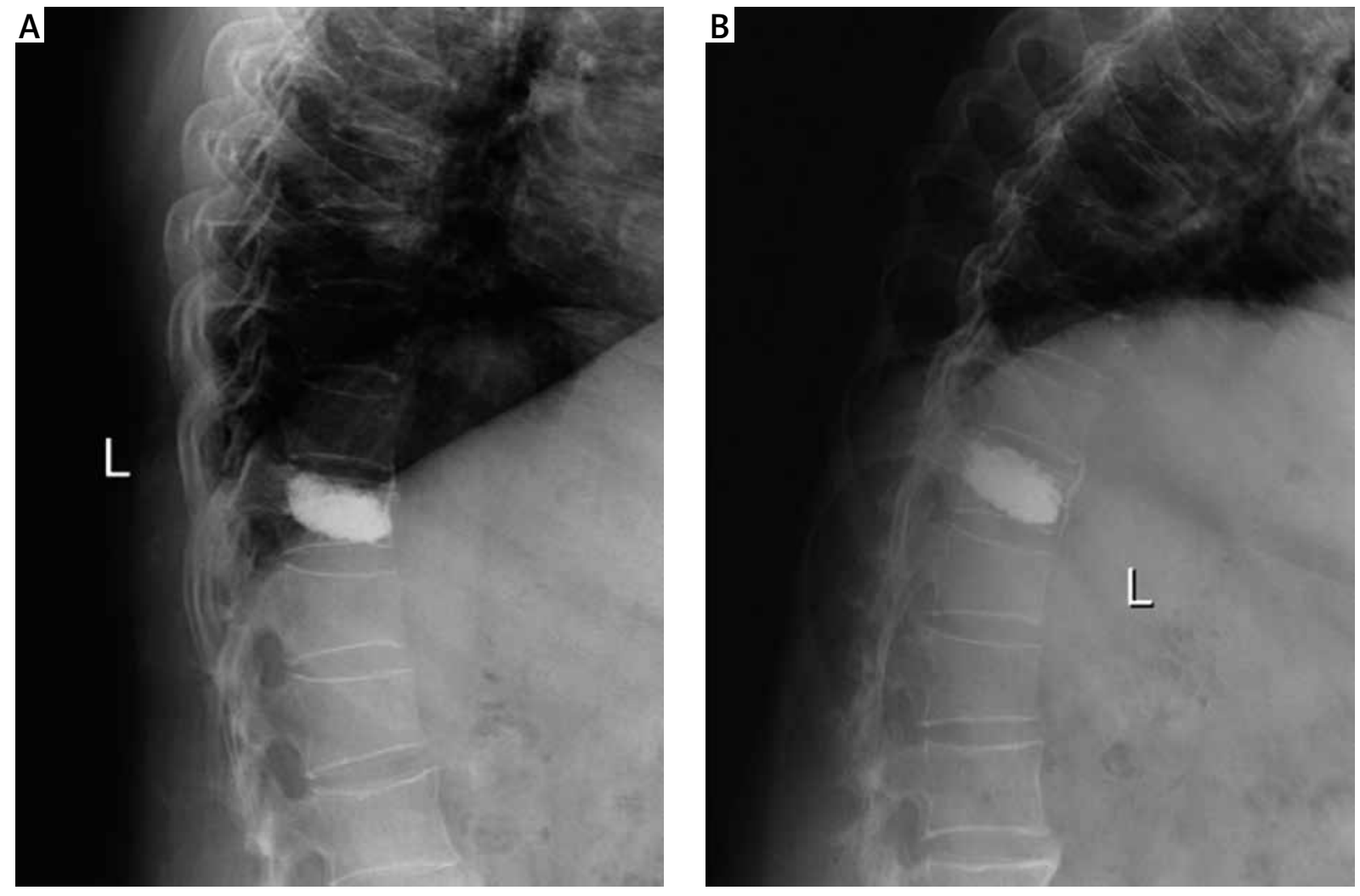

Photo 5. Postoperative dynamic radiographs at 6 months after the operation. No evidence of instability or hypermobility was found at the T10/11 level on postoperative extension (A) and flexion (B) lateral dynamic radiographs 

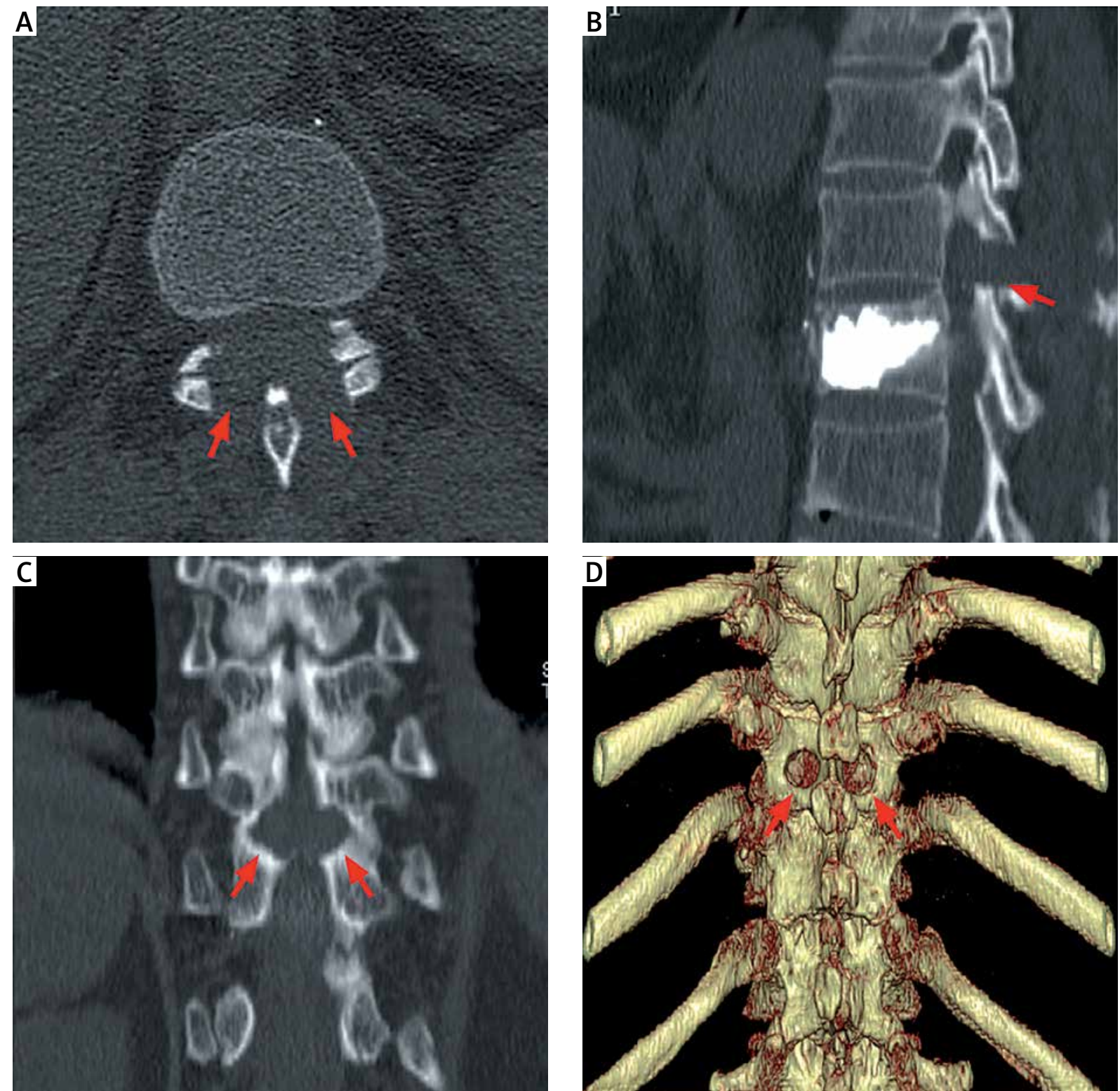

Photo 6. Postoperative CT scan. A - A bilateral translaminar osseous-channel (arrows) was established and the ossified mass (OLF) was completely removed after the $\mathrm{p}$-PELD procedure. A translaminar osseous-channel (arrows) and methyl methacrylate strengthening of T11 were presented on sagittal reconstructed CT scan (B) and coronal reconstructed CT scan (C). D - Bilateral independent translaminar osseous channel (arrows) is observed on 3-dimensional CT reconstruction 

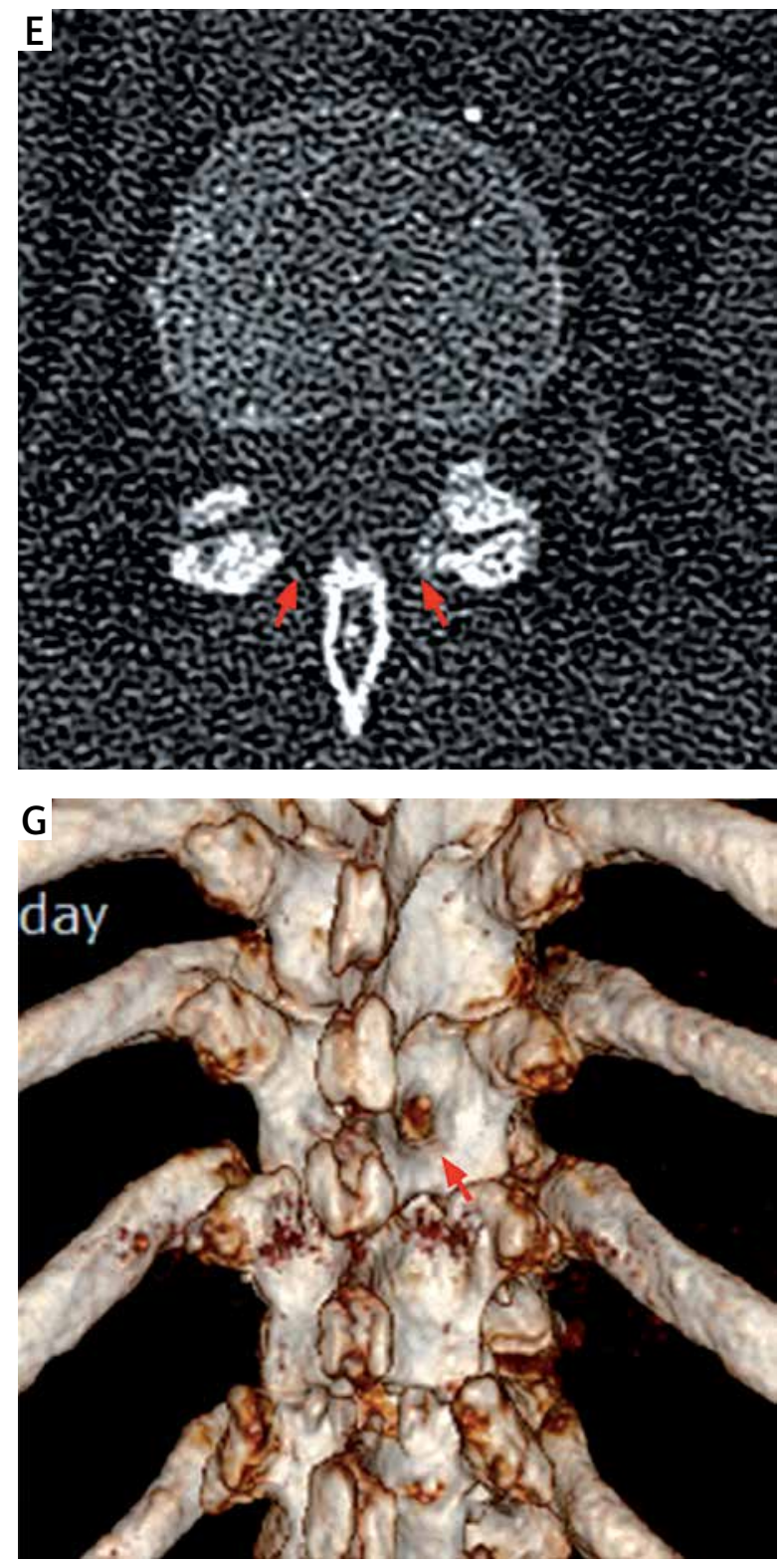

reduce the incidence of this disease. The most commonly involved segments are located at the lower thoracic spine [7], especially the T10-12 level [22, 24]. Multiple symptoms, including radicular and/or myelopathic symptoms, may occur $[25,26]$.

Due to the progressive nature of OLF and the finding that it is refractory to conservative treatment, surgical intervention is recommended once neurological symptoms are present $[5,27]$. On the other hand, surgery is also facilitated to avoid the occurrence of irreversible damage to the spinal cord after minor trauma, which has a pathological basis in the thoracic spinal stenosis caused

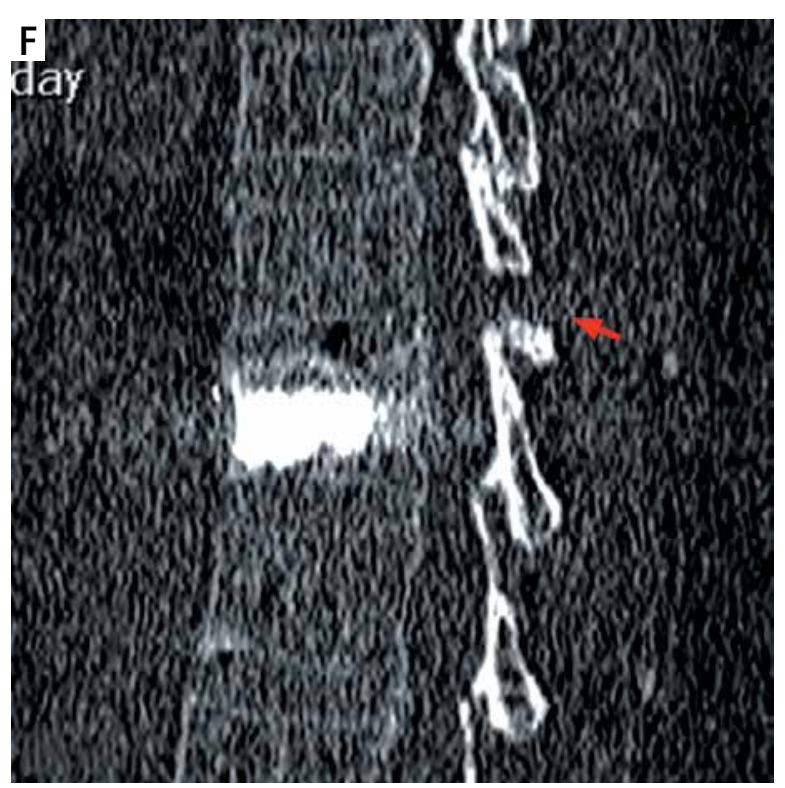

Photo 6. Cont. At 6 months after surgery, CT scans showing a sufficient resection of the ossified mass (OLF) and a reduction of the translaminar osseous channel (arrows) due to the self-repair of bone tissue ( $\mathbf{E}$ - axial view, $\mathbf{F}$ - sagittal view and $\mathbf{G}-3 \mathrm{D}$ view)

by $\operatorname{OLF}[7,28]$. With the goal of developing a surgical intervention able to remove the pathological lesion and to achieve sufficient decompression of the neural structures [7, 29, 30], various surgical techniques have been explored, including posterior and non-posterior approaches through open or non-open methods. Because the neurological deficits are due to the dorsal compression of the spinal cord secondary to OLF [29], most surgeons advocate the use of posterior decompression methods, which include laminectomy [2, 8], laminotomy [20], and laminoplasty [10], as more appropriate for the treatment of thoracic OLF. 

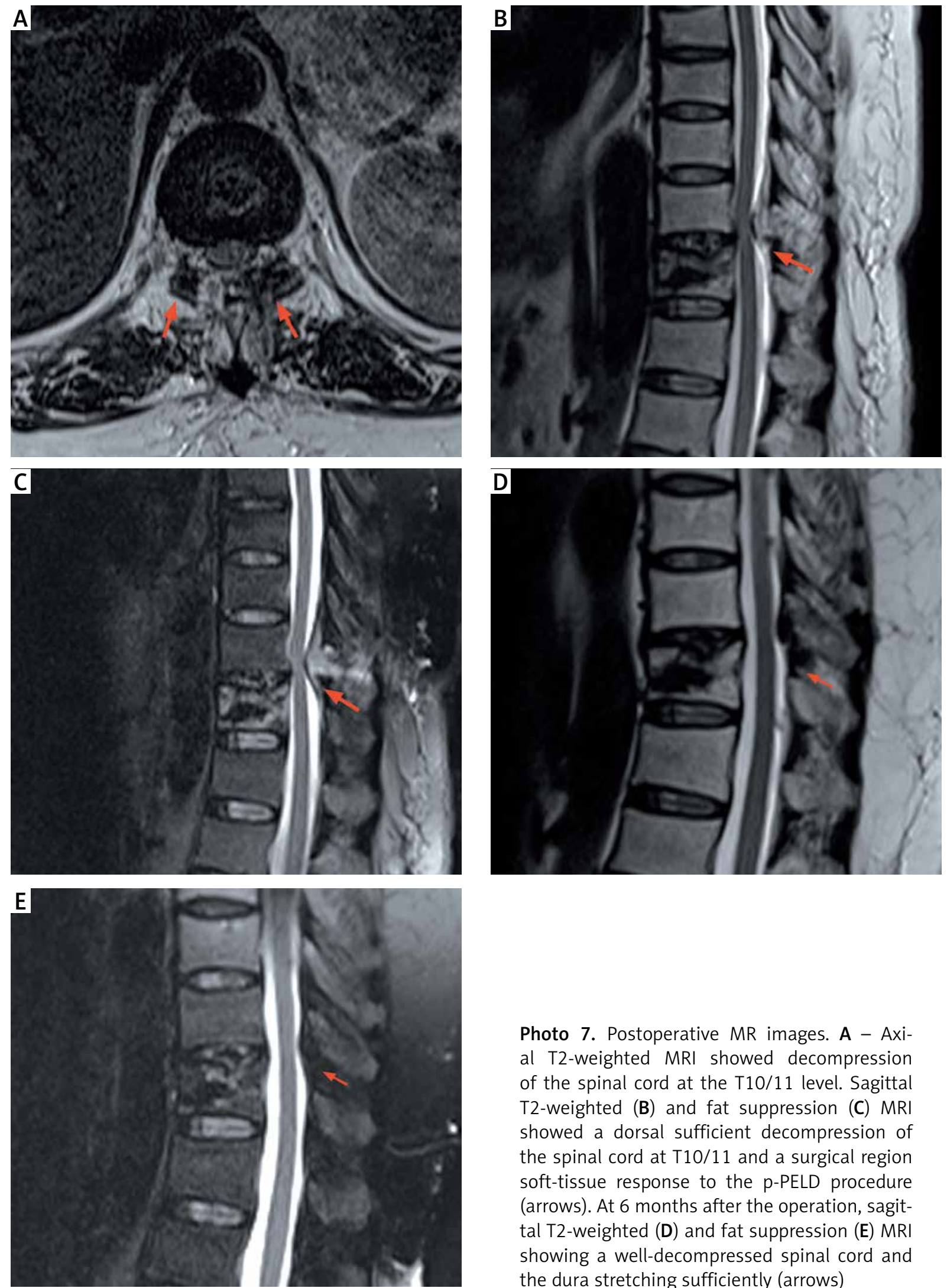

Photo 7. Postoperative MR images. A - Axial T2-weighted MRI showed decompression of the spinal cord at the T10/11 level. Sagittal T2-weighted (B) and fat suppression (C) MRI showed a dorsal sufficient decompression of the spinal cord at T10/11 and a surgical region soft-tissue response to the p-PELD procedure (arrows). At 6 months after the operation, sagittal T2-weighted (D) and fat suppression (E) MRI showing a well-decompressed spinal cord and the dura stretching sufficiently (arrows) 
Table II. Clinical results of the patient before and after surgery

\begin{tabular}{|lcccc|}
\hline Variable & \multicolumn{4}{c|}{ Score } \\
\cline { 2 - 5 } & Preop. & 1 week postop. & 6 months postop. & 2 years postop. \\
\hline mJOA & 2 & 4 & 7 & 10 \\
\hline VAS of thoracolumbar back pain & 6 & 3 & 1 & 0 \\
\hline
\end{tabular}

mJOA - modified Japanese Orthopaedic Association, VAS - visual analogue scale, preop. - preoperative, postop. - postoperative.

Although various surgical procedures have been reported to be effective for OLF in the literature, the optimal surgical approach has not yet been determined [4, 17], and surgical outcomes are not always satisfactory $[9,28]$. The reported mean neurological recovery rate after decompression surgery has varied from $31 \%$ to $68 \%$ in the literature $[29,31]$, the incidence of neurological deterioration after surgery is reported to be as high as $14.5 \%$ to $33 \%$ [32, 33]. and the overall complication rate reported in the literature ranges from $35 \%$ to $51 \%$ [7, 33]. Many scholars consider that one of the reasons for the poor results achieved after decompression surgery is severe iatrogenic injury of the posterior column stable structure, which is related to delayed kyphosis and causes late neurological deterioration [28, 29, 34]. In addition, preservation of the posterior column stable structure is necessary to avoid internal instrumentation and concomitant fusion [10, 35].

To achieve a satisfactory result in decompression surgery while minimizing iatrogenic damage to the spinal stabilizing structures, a minimally invasive and effective surgical technique is warranted [28, 34]. In recent years, the microendoscopic discectomy (MED) technique, combined with the use of a tubular retractor system, has been explored for the treatment of thoracic $\operatorname{OLF}[3,34,36]$. As reported in previous studies $[3,34]$, the mean recovery rate after MED ranges from $49.10 \%$ to $44.9 \%$. Even though good clinical results have been reported with this procedure, there is still a risk concomitant with delayed kyphosis [36], and some authors have stopped performing this procedure because of technical difficulties [36, 37].

Superior to the MED technique, a percutaneous spinal endoscopic procedure is widely applied in cervical and lumbar spine disorders to achieve excellent surgical visualization [15], an enlarged visual field due to the use of optical instruments, less iatrogenic trauma, and a faster postoperative recovery process. With growth of experience among spinal surgeons, improvement in surgical instruments, and the ability to achieve adequate bone and soft tissue resection, the scope of indications for the percutaneous spinal endoscopic technique has expanded [38, 39]. However, few papers have reported the use of percutaneous endoscopic ligamentectomy decompression for TM caused by OLF. Based on our proficiency in percutaneous endoscopic discectomy for cervical and lumbar spine disorders, an attempt was made to use the percutaneous endoscopic technique to treat TM caused by OLF.

In this case, an osseous channel is created in the bilateral lamina to precisely assist p-PELD of the lesion, and the rim of the lamina is left intact both superiorly and inferiorly. During its course, the spinous process, supraspinal ligaments, interspinous ligament and lateral part of the facet joints are preserved, which is important to prevent postoperative segmental instability and delayed kyphosis. Therefore, this procedure achieves satisfactory clinical results through adequate decompression and avoids the economic burden caused by the need for additional internal fixation and fusion. For this patient, who had been treated with a PVP for the inferior vertebral body adjacent to the involved segment, the $\mathrm{p}$-PELD procedure was more appropriate, and no postoperative segmental instability or kyphosis was noted at the 2-year follow-up. Another rationale for using the p-PELD technique for treating thoracic OLF is its excellent ability to provide illumination and visualization of a $30^{\circ}$ angle field, which was used to obtain an enlarged view of the lesions and a sufficiently wide working area to deal with the lesions.

Our technique is different from the interlaminar endoscopic discectomy technique that was reported by Ruetten and Miao [12, 40]. Although they also used spinal endoscopy to decompress the OLF-related TM, there are many differences between their technique and our bilateral translaminar osseous-channel assist p-PELD technique. First, we used translaminar osseous-channel decompression rath- 
er than laminoplasty to preserve the intact rim of the lamina both superiorly and inferiorly, and that will provide more stability and postoperative self-healing for the translaminar osseous tunnel. Second, under the guidance of intraoperative C-arm X-rays, we used a trephine instead of a high-speed drill to punch through the lamina and establish an osseous channel to assist p-PELD, which provides more security for avoiding the frequent stimulation of high-speed drill buffeting and greater efficiency for the overall removal of the laminar excision; furthermore, the entire duration of the operative procedure for bilateral decompression was 109 min. Third, we performed ipsilateral decompression with a bilateral approach rather than bilateral decompression with a uniportal approach because accurate and direct ipsilateral decompression provides greater security and efficiency. After all, the spinal canal in the thoracic region is so narrow that there is very little room for operating [29], and inserting even a fine hook or 1 -mm rongeur into the stenotic thoracic canal may cause irreversible neurological deterioration [2].

Although a satisfactory clinical outcome was achieved in this patient with no serious complications after the p-PELD technique was performed, there are some potential disadvantages of this minimally invasive procedure. We believe that the major disadvantage of the p-PELD procedures is its steep learning curve due to the angled field of two-dimensional visualization and the difficulty of hand-eye coordination. However, those can be overcome by emphasizing training in p-PELD procedures in the lumbar spine. In addition, to reduce the risk of neurological deterioration and improve the safety of surgery, many technical tips for this p-PELD procedure should be noted. One technical tip is to exactly pierce the inner cortex of the lamina by gently and repeatedly rotating the trephine to facilitate the overall removal of the laminar excision. Particular attention should be paid to the trephine, which can be monitored on the intraoperative $\mathrm{X}$-ray to ensure that the inner cortex is penetrated without any violence to the spinal cord, which may cause irreversible neurological deterioration. Another technical tip is to drill the thickest part of the OLF so that it is paper thin, especially in its central part, to avoid detachment and floating of the OLF fragment, which is difficult to fix and is not conducive to subsequent buffing. Subsequently, the periphery of the OLF is drilled so that the paper-thin OLF remains intact to protect the spinal cord from damage. Then, we cautiously dissected and removed the paper-thin OLF fragment away from the dura. In addition, a blunt hook was used to find the medial edges of the pedicles to define the range required for bone resection while remaining conscious that the articular process or laminar debridement should not be less than $50 \%$ so as not to affect spinal stability and thereby minimize the risk of spinal instability or kyphotic deformity caused by the excessive bone resection during the procedure [24].

In this case, the p-PELD procedure achieved satisfactory clinical results with many advantages, such as less iatrogenic invasiveness, excellent illumination and visualization, a reduced economic burden and higher safety. Each procedure has its own indications, including whether it is a minimally invasive procedure or not. As an accurate and controlled decompression technique, this p-PELD procedure is more adapted for localized and isolated lesions than extensive and continual lesions. Therefore, it is more appropriate in patients with single-level OLF-related TM. Cerebrospinal fluid leakage (CFL) is the main complication after thoracic OLF surgery reported in the literature [11]. It was not noted in this case. We speculate that there was no dural ossification in this patient.

We would like to highlight that there are limitations of this study. Only 1 patient was included in the study, limiting our interpretation of the results. Another limitation is that the patient who was included did not have dural ossification, which reduces the difficulty of the p-PELD procedures. Consequently, a larger case study is warranted to verify the efficacy and safety of this p-PELD technique in the treatment of OLF-related TM.

\section{Conclusions}

In this case, we used a novel minimally invasive technique, bilateral translaminar osseous-channel assisted p-PELD for the treatment of OLF-related TM, and satisfactory results were obtained. Theoretically, the p-PELD technique has many advantages, such as less iatrogenic trauma, excellent illumination and visualization, a particular operating field, and no need for internal fixation; furthermore, it is technically feasible for the treatment of single-level and isolated OLF-related TM. Definitive therapeutic effects and safety still need to be demonstrated in a larger sample clinical study, but this P-PELD technique 
should be considered as an optional treatment for OLF-related TM due to its potential indications and advantages.

\section{Acknowledgments}

We thank Fujun Wu, Xing Chen, Hao Yuan, and Jin Li for the preparation of illustrations.

\section{Conflict of interest}

\section{The authors declare no conflict of interest.}

\section{References}

1. Miura K, Koda M, Abe T, et al. Thoracic myelopathy caused by calcification of the ligamentum flavum. J Rural Med 2020; 15: 65-7.

2. Wang T, Yin C, Wang D, et al. Surgical technique for decompression of severe thoracic myelopathy due to tuberous ossification of ligamentum flavum. Clin Spine Surg 2017; 30: E7-12.

3. Baba S, Oshima Y, Iwahori T, et al. Microendoscopic posterior decompression for the treatment of thoracic myelopathy caused by ossification of the ligamentum flavum: a technical report. Eur Spine J 2016; 25: 1912-9.

4. Ahn DK, Lee S, Moon SH, et al. Ossification of the ligamentum flavum. Asian Spine J 2014; 8: 89-96.

5. Osman NS, Cheung ZB, Hussain AK, et al. Outcomes and complications following laminectomy alone for thoracic myelopathy due to ossified ligamentum flavum: a systematic review and meta-analysis. Spine (Phila Pa 1976), 2018; 43: E842-e8.

6. Ando K, Imagama S, Kaito T, et al. Outcomes of surgery for thoracic myelopathy owing to thoracic ossification of the ligamentum flavum in a nationwide multicenter prospectively collected study in 223 patients: is instrumented fusion necessary? Spine (Phila Pa 1976) 2020; 45: E170-e8.

7. Li Z, Ren D, Zhao Y, et al. Clinical characteristics and surgical outcome of thoracic myelopathy caused by ossification of the ligamentum flavum: a retrospective analysis of 85 cases. Spinal Cord 2016; 54: 188-96.

8. Jia LS, Chen XS, Zhou SY, et al. En bloc resection of lamina and ossified ligamentum flavum in the treatment of thoracic ossification of the ligamentum flavum. Neurosurgery 2010; 66: 1181-6.

9. Wang H, Wei F, Long H, et al. Surgical outcome of thoracic myelopathy caused by ossification of ligamentum flavum. J Clin Neurosci 2017; 45: 83-8.

10. Wang VY, Kanter AS, Mummaneni PV. Removal of ossified lig amentum flavum via a minimally invasive surgical approach. Neurosurg Focus 2008; 25: E7.

11. Hou X, Chen Z, Sun C, et al. A systematic review of complications in thoracic spine surgery for ossification of ligamentum flavum. Spinal Cord 2018; 56: 301-7.

12. Ruetten S, Hahn P, Oezdemir S, et al. Full-endoscopic uniportal decompression in disc herniations and stenosis of the thoracic spine using the interlaminar, extraforaminal, or transthoracic retropleural approach. J Neurosurg Spine 2018; 29: 157-68.
13. Du Q, Wang X, Qin JP, et al. Percutaneous full-endoscopic anterior transcorporeal procedure for cervical disc herniation: a novel procedure and early follow-up study. World Neurosurg 2018; 112: e23-30.

14. Iwai $\mathrm{H}$, Inanami $\mathrm{H}$, Koga $\mathrm{H}$. Full-endoscopic spine surgery for the treatment of lumbar ossification of the ligamentum flavum: technical report. World Neurosurg 2020; 142: 487-94.e1.

15. Shu W, Zhu H, Liu R, et al. Posterior percutaneous endoscopic cervical foraminotomy and discectomy for degenerative cervical radiculopathy using intraoperative O-arm imaging. Videosurgery Miniinv 2019; 14: 551-9.

16. Chapman CR, Casey KL, Dubner R, et al. Pain measurement: an overview. Pain 1985; 22: 1-31.

17. Wang T, Du C, Zheng X, et al. Surgical strategies for thoracic myelopathy due to ossification of ligamentum flavum: a technical note based on radiological type. Turk Neurosurg 2017,doi: 10.5137/1019-5149.jtn.20391-17.1.

18. Feng FB, Sun CG, Chen ZQ. Progress on clinical characteristics and identification of location of thoracic ossification of the ligamentum flavum. Orthop Surg 2015; 7: 87-96.

19. Guo JJ, Luk KD, Karppinen J, et al. Prevalence, distribution, and morphology of ossification of the ligamentum flavum: a population study of one thousand seven hundred thirty-six magnetic resonance imaging scans. Spine (Phila Pa 1976) 2010; 35: 51-6.

20. Aizawa T, Sato T, Sasaki H, et al. Thoracic myelopathy caused by ossification of the ligamentum flavum: clinical features and surgical results in the Japanese population. J Neurosurg Spine 2006; 5: 514-9.

21. Lang N, Yuan HS, Wang HL, et al. Epidemiological survey of ossification of the ligamentum flavum in thoracic spine: CT imaging observation of 993 cases. Eur Spine J 2013; 22: 857-62.

22. Kim SI, Ha KY, Lee JW, et al. Prevalence and related clinical factors of thoracic ossification of the ligamentum flavum-a computed tomography-based cross-sectional study. Spine J 2018; 18: 551-7.

23. Li B, Guo S, Qiu G, et al. A potential mechanism of dural ossification in ossification of ligamentum flavum. Med Hypotheses 2016; 92: 1-2.

24. Xiaobing Z, Xingchen L, Honggang Z, et al. "U" route transforaminal percutaneous endoscopic thoracic discectomy as a new treatment for thoracic spinal stenosis. Int Orthop 2018; 43: 825-32.

25. Roelz R, Scholz C, Klingler JH, et al. Giant central thoracic disc herniations: surgical outcome in 17 consecutive patients treated by mini-thoracotomy. Eur Spine J 2016; 25: 1443-51.

26. Lee BJ, Park JH, Jeon SR, et al. Clinically significant radiographic parameter for thoracic myelopathy caused by ossification of the ligamentum flavum. Eur Spine J 2019; 28: 1846-54.

27. Li B, Qiu G, Guo S, et al. Dural ossification associated with ossification of ligamentum flavum in the thoracic spine: a retrospective analysis. BMJ Open 2016; 6: e013887.

28. Eun SS, Kumar R, Choi WG, et al. Lamina fenestration technique for treatment of thoracic ossified ligamentum flavum: 2-year follow-up result. J Neurol Surg A Cent Eur Neurosurg 2017; 78: 286-90.

29. Zhong ZM, Wu Q, Meng TT, et al. Clinical outcomes after decompressive laminectomy for symptomatic ossification of lig- 
amentum flavum at the thoracic spine. J Clin Neurosci 2016; 28: 77-81.

30. Ruetten S, Hahn P, Oezdemir S, et al. Decompression of the anterior thoracic spinal canal using a novel full-endoscopic uniportal transthoracic retropleural technique-an anatomical feasibility study in human cadavers. Clin Anat 2018; 31: 716-23.

31. Nie ZH, Liu FJ, Shen Y, et al. Lamina osteotomy and replantation with miniplate fixation for thoracic myelopathy due to ossification of the ligamentum flavum. Orthopedics 2013; 36: e353-9.

32. Young WF, Baron E. Acute neurologic deterioration after surgical treatment for thoracic spinal stenosis. J Clin Neurosci 2001; 8: 129-32.

33. Yamazaki M, Koda M, Okawa A, et al. Transient paraparesis after laminectomy for thoracic ossification of the posterior longitudinal ligament and ossification of the ligamentum flavum. Spinal Cord 2006; 44: 130-4.

34. Zhao W, Shen C, Cai R, et al. Minimally invasive surgery for resection of ossification of the ligamentum flavum in the thoracic spine. VIdeosurgery Miniinv 2017; 12: 96-105.

35. Kawaguchi Y, Yasuda T, Seki S, et al. Variables affecting postsurgical prognosis of thoracic myelopathy caused by ossification of the ligamentum flavum. Spine J 2013; 13: 1095-107.

36. Ikuta K, Tarukado K, Senba H, et al. Decompression procedure using a microendoscopic technique for thoracic myelopathy caused by ossification of the ligamentum flavum. Minim Invasive Neurosurg 2011; 54: 271-3.

37. Martin-Laez R, Martinez-Agueros JA, Suarez-Fernandez D, et al. Complications of endoscopic microdiscectomy using the EASY$\mathrm{GO}$ ! system: is there any difference with conventional discectomy during the learning-curve period? Acta Neurochir 2012; 154: 1023-32.

38. Ruetten S, Komp M, Merk H, et al. Full-endoscopic anterior decompression versus conventional anterior decompression and fusion in cervical disc herniations. Int Orthop 2009; 33: 1677-82.

39. Ruetten S, Komp M, Merk H, et al. Recurrent lumbar disc herniation after conventional discectomy: a prospective, randomized study comparing full-endoscopic interlaminar and transforaminal versus microsurgical revision. J Spinal Disord Tech 2009; 22: 122-9.

40. Miao X, He D, Wu T, et al. Percutaneous endoscopic spine minimally invasive technique for decompression therapy of thoracic myelopathy caused by ossification of the ligamentum flavum. World Neurosurg 2018; 114: 8-12.

Received: 31.05 .2020 , accepted: 20.09 .2020 